\title{
Sero prevalence of syphilis infection among patients attending Antenatal Care \& Sexually transmitted disease (STD) clinics: observations from a tertiary care hospital of northern India
}

\author{
Nazish Fatima*, Abida Malik, Parvez Anwar Khan, Sana Ali, Haris Manzoor Khan, Nabeela \\ Immunology section, Dept of Microbiology, Jawaharlal Nehru Medical College, Aligarh Muslim University, Aligarh, India \\ Email address: \\ nazsham28@gmail.com (N. Fatima)
}

To cite this article:

Nazish Fatima, Abida Malik, Parvez Anwar Khan, Sana Ali, Haris Manzoor Khan, Nabeela. Sero Prevalence of Syphilis Infection among Patients Attending Antenatal Care \& Sexually Transmitted Disease (STD) Clinics: Observations from a Tertiary Care Hospital of Northern India. American Journal of Internal Medicine. Vol. 2, No. 1, 2014, pp. 6-9. doi: 10.11648/j.ajim.20140201.12

\begin{abstract}
Background:- Syphilis generally considered to be a sexually transmitted disease, can also be transmitted in utero, and rarely by blood transfusion or non-sexual contact and is characterized by episodes of active disease, interrupted by latency. Congenital syphilis poses a significant challenge especially because infants may be still born, asymptomatic or present with a variable clinical picture at birth. Syphilis has been implicated in increasing susceptibility to HIV infection. We under took this study to determine the seroprevalence of syphilis in a tertiary care setting of Northern India and to identify an association with HIV-infection. Material \& Method: - Of the total 19860 samples received during 5 years, VDRL was performed on 19860 samples and TPHA on 65 samples. Results: - $65(0.33 \%)$ were found to be positive by VDRL. The patient's positive for syphilis included 23 pregnant females $(0.15 \%$ of the ANC samples received), $14(21.5 \%)$ HIV positive patients, 24 were from STD clinic (2.3\% of the samples received from STD clinic). Conclusions: - The strong association between syphilis and HIV seroprevelence in this study suggests that acceleration of direct linkages between STI testing and HIV counseling and testing will be useful in enhancing the control of STI and HIV in India.
\end{abstract}

Keywords: Syphilis, VDRL, TPHA, HIV, ANC

\section{Introduction}

Sexually transmitted infections (STIs) are some of the most common causes of illness worldwide. Incidence of STIs, in the world, is rising despite improved methods of diagnosis and treatment [1].World over, excluding HIV, there are 333 million new cases of STIs per year. Sexually transmitted infections are far more common in developing countries than industrial countries [2].

Syphilis is a chronic systemic infection caused by the spirochaete,"Treponema palladium". Generally considered to be a sexually transmitted disease, this infection can also be transmitted in utero, and rarely by blood transfusion or non-sexual contact [3] and is characterized by episodes of active disease, interrupted by latency. Comprehensive data on the prevalence of syphilis is not available in most of the developing countries. There is high variability in the seroprevalence rates among different socioeconomic groups in these countries $[4,5]$. Seroprevalence rates in India range from $9.07 \%$ among high risk STI patients in a study from Himachal Pradesh to $21.9 \%$ in long distance truck drivers in central India [6] and as low as $1.9 \%$ among ANC attendees in a study by $\mathrm{WHO}[7]$.

Congenital syphilis poses a significant challenge especially because infants may be still born, asymptomatic at birth but nonetheless infected, or present with a highly variable clinical picture, thereby precluding easy clinical diagnosis. The high risk of congenital syphilis in untreated or inadequately treated mothers (4\%) is one reason why $40 \%$ of these pregnancies end in fetal loss or perinatal death [8].

The most widely used screening tests for syphilis are the Venereal Disease Research Laboratories (VDRL) and the rapid plasma reagin (RPR) tests. As these tests employ cardiolipin antigen, biological false positive results may occur in many cases [10] Thus, a confirmatory test for the detection of T. pallidum antibodies, such as the T. pallidum hemagglutination assay (TPHA) [11, 12] or fluorescent treponemal antibody adsorption test is required [13]. 
Concurrent infection with Treponema pallidum and HIV is posing as a serious health problem. It has been found that syphilis infection increases susceptibility to HIV infection [14]. Due to immunosuppression HIV co infection alters the natural history of syphilis and response to treatment. There has been an increase in case of neurosyphilis among the HIV infected persons, even after complete recommended treatment [15].

In view of the above background, we under took this study to determine the seroprevalence of syphilis in a tertiary care setting of Northern India and to identify an association with HIV-infection.

\section{Material and Methods}

A retrospective study was conducted over a period of 5 years in the Department of Microbiology, Jawaharlal Nehru Medical College, Aligarh Muslim University, Aligarh. The study included 19860 samples received during 2009- 2013 of which 17440 were from ANC patients. VDRL was performed on 19860 samples and TPHA on 65 samples.

VDRL: Qualitative and quantitative VDRL tests were carried out on all sera using VDRL antigen prepared by the Institute of Serology, Calcutta. Sera showing no clump were reported as non-reactive. Samples showing small clumps were considered weakly reactive. Those with medium sized or large clumps were reported as reactive. Quantitative test was performed on all reactive sera including those showing a weak reaction. The results were reported in terms of the highest dilution which gave a frank positive reaction. Both reactive and weakly reactive sera were labeled as positive.

TPHA was performed by TPHA kit (TPHA Emoagglutinazione in micropiastra) supplied by GIESSE diagnostics as per manufacturer's instructions.

\section{Results}

Out of 19860 samples on which VDRL was performed $65(0.33 \%)$ were found to be positive. These were further confirmed by TPHA and 59 (91\%) out of 65 samples were positive. A total agreement was seen between TPHA and VDRL with a titre of 1 in 16 and above while in as many as $9 \%$ sera with VDRL titres $\leq 1$ in 8 , TPHA was negative indicating that titres above 1 in 8 should be considered as true reactive to minimize chances of biologically false positive reactions. Among VDRL reactive patients, $36(55.4 \%)$ were males and 29(44.6\%) females. Age wise distribution was $6(9.2 \%)$ patients in age group $10-20$ years, $36(55.4 \%)$ in $20-30$ years, $17(26.1 \%)$ in age group 30 40years, $4(6.15 \%)$ in 40-50 years and $2(3.07 \%)$ were $>50$ years. When HIV status of these patients was studied it was found that 6 patients were positive for HIV in 2008, 2 were positive in 2009, 3 in 2010 and 3 were positive in 2011. The patients positive for syphilis included 23 pregnant females $(0.15 \%$ of the ANC samples received), $14(21.5 \%)$ HIV positive patients, 24 were from STD clinic $(2.3 \%$ of the samples received from STD clinic).

\section{Discussion}

Comprehensive data on the incidence of syphilis is not available from most developing countries. Serological surveys continue to be the best source of information on the prevalence of syphilis. Minimal estimates of yearly incidence of syphilis are 12 million worldwide [16].

Estimates reveal that South Asia has the highest number of syphilis cases in the world [7]. Most of the international studies on seroprevalence of syphilis range between $6-23 \%$. A study conducted in three major cities of Russia (Moscow Volgograd and Barman) by Rhodes et al showed seroprevalence of syphilis to be $8 \%, 20 \%$ and $6 \%$ while that of HIV was $14 \%, 3 \%$ and $9 \%$ respectively, $[17,18]$. Studies conducted in India, Bangladesh and Germany showed a seroprevalence rate of syphilis in Intravenous Drug Users (IDU) to be $6.07 \%, 23 \%$ and $33 \%$ respectively. The possible explanation for the difference could be the difference in sub groups of population studies and the diagnostic test employed in addition to the region, gender, ethnic and socio-economic factors. Accurate figures on the incidence of STDs are difficult to obtain not only because of inadequate reporting but because of the secrecy that surrounds them. All available data, however indicate a high prevalence of STD (from 1\%-14\%) in the vulnerable population groups [19]).

By comparing international studies with our study on seroprevalence of syphilis, our results are quite low. VDRL was positive in $0.262 \%$ while TPHA in $0.236 \%$. The difference could be attributed to biological false positives (9.61\%) especially in pregnant females and low titre VDRL positives. Hence, TPHA is a usefull test in confirming true positive syphilis cases.

Prevalence was found to be slightly higher in men than in women (table-1).Prevalence was highest among pregnant females followed by HIV positives and patients from STD clinic. High prevalence among pregnant females could be explained on the basis of large proportion of samples included in the study from the antenatal clinic (table-3).

Table1. Sex wise distribution of Syphilis Patients

\begin{tabular}{cl}
\hline MALES & $\mathbf{3 6}(\mathbf{5 5 . 4 \% )}$ \\
\hline FEMALES & $29(44.6 \%)$ \\
\hline
\end{tabular}

Table 2. Age wise distribution of Syphilis Patients

\begin{tabular}{ccc}
\hline AGE GROUP (yrs) & NO. OF PATIENTS & $\begin{array}{c}\text { PERCENTAGE OF } \\
\text { PATIENTS }\end{array}$ \\
\hline $10-20$ & 6 & $9.2 \%$ \\
$20-30$ & 36 & $55.4 \%$ \\
$30-40$ & 17 & $26.1 \%$ \\
$40-50$ & 4 & $6.15 \%$ \\
$>50$ & 2 & $3.07 \%$ \\
\hline
\end{tabular}


Table 3. Clinical presentation of Syphilis Patients

\begin{tabular}{cc}
\hline Types of patients & Percentage \\
\hline ANC & $23(0.15 \%)$ \\
STD & $24(2.3 \%)$ \\
HIV Positive & $14(21.5 \%)$ \\
\hline
\end{tabular}

Prevalence was also highest among the age group 20-30 years, which could be due to increased risk of exposure in this group and most of pregnant females which constituted a large section of study group also fall in this category (table-2).

In India the prevalence of syphilis ranges between 2.0$4.8 \%$ among women of reproductive age [20, 21, 22, 23]. The $0.15 \%$ seroprevalence rate for syphilis among ANC attendees in our study is quiet low as compared to other studies [7]. A similar low seroprevalence rate of syphilis $0.9 \%, 0.24 \%, 1 \%$ was found among ANC attendees in a study conducted at Pakistan, Burkina Faso and Ethiopia respectively $[24,25,26]$. This low seroprevalence of syphilis may be due to over-the-counter use of antibiotics for minor complaints and the easy availability of drugs in the countries. The limitation of our study was the absence of data from rural areas. Also, the study was hospital based and not population based. Gestational syphilis is of particular concern in under equipped health systems and wherever access to health care is a major limiting factor for any STD programme effectiveness.

Among the STD clinic attendees, seroprevalence of syphilis was found to be $2.3 \%$. The incidence of syphilis measured in a population based cohort in Tanzania revealed a rate of $1.8 \%$ with slightly higher rates in men [27]. Similar incidence rates were seen in a multicenterd study at San Francisco (1.5\%) and among female sex workers in Mexico $(2.4 \%)$ [28,29]. Our findings were similar $(1.75 \%)$ to a population based study conducted in A.P., India (30). However, another study in Karnataka revealed a much lower sero prevalence of syphilis (0.4\%) [31]. Contrasting this, a high sero-prevalence rate among STI patients was seen in a study in H.P. (9.07\%) [6].The heterogeneous nature of STI epidemic in India explains the differences in findings at the district level. Programmatic prevention and treatment programs need to be started which answer to the local conditions.

Observing the HIV-syphilis co-seroprevalence, we found that $21.5 \%$ of sero positive syphilis cases were also HIV infected. Similar HIV- syphilis co-prevalence rates were seen in a population based study in Andhra Pradesh, India (23\%) [30].This finding has important implications for STI syndromic management and HIV testing strategies being currently followed in India.

Evidence that STIs may facilitate HIV infection has focused attention to the situation. The biological explanation for enhanced susceptibility to HIV among individuals infected with syphilis is based on the theory that the break down in mucosal integrity due to ulceration provides a portal of entry for the HIV virus. In addition, this ulceration may result in an influx of CD4 lymphocytes locally increasing the number of HIV target cells which has been observed in the setting of chancroid and recurrent genital herpes infections [32,33,34]. Syphilis may also increase HIV infectiousness and transmission among HIV sero-positive individuals dually infected with syphilis by increasing the amount of HIV viral shedding [35,36,37,38]. We propose that the increased risk of HIV-1 infection among patients with incident syphilis in this study probably represents a combination of both the effects of syphilis on suspects among HIV-1 infected patients and also an effect on transmission with individuals being exposed to dually infected (Both HIV-1 and syphilis) partners and acquiring both infections simultaneously.

\section{Conclusions}

The low prevalence of syphilis among pregnant women and adult general population is very encouraging. But resources need to be continuously dedicated towards STI \& HIV control programs.

Public health interventions to promote awareness of syphilis among physicians and populations at risk in India are urgently needed to avoid the adverse consequences which could result from missed diagnosis or improper treatment. In addition to contributing to the spread of HIV in India, untreated syphilis could also contribute to poor health outcomes resulting from the consequences of latent stages of the disease and maternal infant transmission with resultant congenital syphilis.

The strong association between syphilis and HIV seroprevelence in this study suggests that acceleration of direct linkages between STI testing and HIV counseling and testing will be useful in enhancing the control of STI and HIV in India.

\section{Acknowledgement}

Special thanks to Mr. Sanjay Sharma for excellent technical expertise.

\section{References}

[1] Hashwani S, Hinan T, Fatima M. Awareness of sexually ransmitted diseases in a selected sample in Karachi. J Pak Med Assoc 1999; 49: 161-4.

[2] Faiz NR, Malik L, Raziq F et al. Frequency of vaginal discharge and its association with various sexually transmitted diseases in women attending antenatal clinic. J Postgrad Med Institute 2005; 19: 86-95.

[3] Goh BT. Syphilis in adults. Sex Transm Infect. 2005; 81:448-52.

[4] Aral S O, Holmess KK. Sexually transmitted disease in the Aids era. Sci Am 1991. 26462-69.69.

[5] World Health Organization An overview of selected curable STDs. Syphilis estimates, 1995. WHO office of HIV/AIDS and STDs. Geneva : WHO, 1995. 
[6] Gawande A V, Vasudeo ND, Zodpey SP. et al Sexualley transmitted in long distance truck drivers. J Commun Dis 2000. $32212-215.215$.

[7] World Health Organization An overview of selected curable STDs. Syphilis estimates, 2001. WHO office of HIV/AIDS and STDs. Geneva : WHO, 2001.

[8] CDC Congenital syphilis -- New York City, 19861988. MMWR. 1989; 38: 825-9.

[9] Lowhagen GB. Syphilis: test procedures and therapeutic strategies. Semin Dermatol. 1990;9:152-9.

[10] Wiwanitkit V. Biological false reactive VDRL tests: when to re-test? Southeast Asian J Trop Med Public Health. 2002;33 (Suppl 3):131-2.

[11] Pope, V., M. B. Fears, W. E. Morrill, A. Castro, and S. E. Kikkert. 2000. Comparison of the Serodia Treponema pallidum particle agglutination, Captia Syphilis-G, and SpiroTeck Reagin II tests with standard test techniques for diagnosis of syphilis. J. Clin. Microbiol. 38:2543-2545.

[12] Rathlav, T. 1967. Hemagglutination tests utilizing antigens from pathogenic and apathogenic Treponema pallidum. Br. J. Vener. Dis. 43:181-185

[13] Hunter, E. F., W. E. Deacon, and P. E. Meyer. 1964. An improved FTA test for syphilis, the absorption procedure (FTA-ABS). Public Health Rep. 79:410-412.

[14] Buchacz K, Patel P, Taylor M, Kerndt PR, Byers RH, Holmberg SD, Klausner JD: Syphilis increases HIV viral load and decreases CD4 cell counts in HIV-infected patients with new syphilis infections. Aids 2004, 18(15):2075-2079

[15] Musher DM. Syphilis, neurosyphilis, penicillin and AIDS. J Infect Dis 1991; 163:1201-6.

[16] K.Park.Preventive and Social Medicine $21^{\text {st }}$ edition. Chapter sexually transmitted diseases p304 2011.

[17] Baveja UK, Chattopadhya D, Khera P, Joshi PL. A cross sectional study of coinfection of hepatitis B virus, hepatitis $\mathrm{C}$ virus and human immunodeficiency amongst a cohort of IDUs at Delhi. Indian J Med Microbiol 2003; 21: 280-3.

[18] Scherbaum N, Baune BT, Micolajezyk R, Kuhlmann T, Reymann G, Reker M. Prevalence and risk factors among drug addicts. BMC Infect Dis 2005; 5: 33-8.

[19] World Health Organization 1992 .Global health situation and projections.Geneva, WHO 1992.

[20] Panda S, Kumar MS, Saravanamurthy PS, et al. sexually transmitted infections and sexual practices in injection drug users and their regular sex partners in Chennai, India. Sex Transm Dis. 2007; 34:250-3.

[21] Carey MP, Ravi V, Chandra PS, et al. Prevalence of HIV, Hepatitis B, syphilis, and chlamydia among adults seeking treatment for a mental disorder in southern India. AIDS Behav. 2007; 11:289-97.

[22] Bhalla P, Chawla R, Garg S, et al. Prevalence of bacterial vaginosis among women in Delhi, India. Indian $\mathrm{J}$ Med Res. 2007; 125:167-72.

[23] Chawla R, Bhalla P, Garg S, et al. Community based study on sero-prevalence of syphilis in New Delhi (India). J Commun Dis. 2004; 36:205-11.
[24] Sharaf Ali Shah, Ghazala Usman et al. Prevelence of syphilis among antenatal clinic attendees in Karachi: Imperative to begin universal screening in Pakistan. JPMA, $2011: 993-7$.

[25] Sombie I, Meda N, Cartoux M, et al.(2000): seroprevelence of Syphilis among womwen attending urban antenatal clinics in Burkina Faso, 1995-8. The DITARME study group Diminunation de la Tranmission Mere-Enfant Sex. Transm. Infect. 77, 37-45.

[26] Andargachew Mulu, Afework Kassu et al. Seroprevelence of Syphilis and HIV-1 during pregnancy in a teaching hospital in Northwest Ethopia. Jpn. J. Infect. Dis., 2007, 60,193-195.

[27] Todd J, Munguti K, Grosskurth H. et al Risk factors for active syphilis and TPHA seroconversion in a rural African population. Sex Transm Infect 2001. 7737-45.45.

[28] Siegel D, Larsen S A, Golden E. et al Prevalence, incidence, and correlates of syphilis seroreactivity in multiethnic San Francisco neighborhoods. Ann Epidemio 11994. 4460-465. 465 .

[29] Uribe-Salas F, Rio-Chiriboga C, Conde-Glez C J. et al Prevalence, incidence, and determinants of syphilis in female commercial sex workers in Mexico City. Sex Transm Dis 1996. 23120-126.126.

[30] Jhon A Schneider, Vemu Laxmi et al. Population- based seroprevelence of HSV-2 and syphilis in Andhra Pradesh state of India. BMC Inf. Dis 2010.

[31] Aalia Hameed, Mateen Izhar, Nafisa Fatima. Seroprevalence of Syphilis in Injecting Drug Users.

[32] Cunningham A L, Turner R R, Miller A C. et al Evolution of recurrent herpes simplex lesions. An immunohistologic study. J Clin Invest 1985. 75226-233.233

[33] Koelle D M, Abbo H, Peck A. et al Direct recovery of herpes simplex virus ()-specific T lymphocyte clones from recurrent genital -2 lesions. J Infect Dis 1994.169956961.961.

[34] Spinola S M, Orazi A, Arno J N. et al Haemophilus ducreyi elicits a cutaneous infiltrate of CD4 cells during experimental human infection. J Infect Dis 1996.173394-402.402.

[35] Ghys P D, Fransen K, Diallo M O. et al The associations between cervicovaginal HIV shedding, sexually transmitted diseases and immunosuppression in female sex workers in Abidjan, Cote d'Ivoire. AIDS 1997. 11F85-F93.F93.

[36] Gadkari D A, Quinn T C, Gangakhedkar R R. et al HIV-1 DNA shedding in genital ulcers and its associated risk factors in Pune, India. J Acquir Immune Defic Syndr Hum Retrovirol 1998. 18277-281.281.

[37] Mbopi-Keou F X, Gresenguet G, Mayaud P. et al Interactions between herpes simplex virus type 2 and human immunodeficiency virus type 1 infection in African women: opportunities for intervention. J Infect Dis 2000. 1821090-1096.1096.

[38] Schacker T, Ryncarz A J, Goddard J. et al Frequent recovery of HIV-1 from genital herpes simplex virus lesions in HIV-1-infected men. JAMA 1998. 28061-66. 